\title{
Linear Tailored Gain Broad Area Semiconductor Lasers
}

\author{
CHRISTOPHER P. LINDSEY, DAVID MEHUYS, STUDENT MEMBER, IEEE, \\ AND AMNON YARIV, FELLOW, IEEE
}

\begin{abstract}
Tailored gain semiconductor lasers capable of high-power operation with single-lobed, nearly diffraction limited beamwidths only a few degrees wide have been demonstrated in proton implanted chirped arrays and "halftone" broad area lasers. We analyze lasers with a linear gain gradient, and obtain analytic approximations for their unsaturated optical eigenmodes. Unlike a uniform array, the fundamental mode of a linear tailored gain laser is the lasing mode at threshold. Mode discrimination may be controlled by varying the spatial gain gradient. All modes of asymmetric tailored gain waveguides have single-lobed far-field patterns offset from $0^{\circ}$. Finally, we utilize tailored gain broad area lasers to make a measurement of the antiguiding parameter, and find $b=\mathbf{2 . 5} \pm \mathbf{0 . 5}$, in agreement with previous results.
\end{abstract}

\section{INTRODUCTION}

$\mathrm{M}$ ANY applications of semiconductor lasers require high optical power outputs. Although this may be achieved in a broad area laser, the beam patterns of conventional devices with uniform spatial gain profiles are very wide, highly irregular, and unstable, and are thus unsuitable for the majority of applications which require a clean, single-lobed optical beam. The undesirable farfield patterns result from the lasing of uncontrolled filaments and higher order lateral modes. Filamentation results from a nonlinear interaction between the optical field and the carrier distribution [1], while the lateral mode problem results from the lasing of higher order modes which have multilobed far-field patterns. The conventional solution to both these problems is to limit the width of the laser stripe to about $10 \mu \mathrm{m}$. Unfortunately, this then limits the maximum power output and minimum beamwidth of the device.

One promising method of obtaining high-power operation and narrow beamwidths is to fabricate a phase-locked laser array by placing many lasers in close proximity. Although the array structure solves the filamentation problem by confining the filaments to individual laser channels, the lateral mode problem remains. The well-known undesirable twin-lobed far-field pattern of a uniformly spaced array of identical lasers [2]-[4] comes about because the lossy interchannel regions in the uniform array

Manuscript received July 30, 1986; revised February 16, 1987. The research described in this paper was performed by the Department of Applied Physics, California Institute of Technology, under contracts with the Office of Naval Research and the National Science Foundation.

C. P. Lindsey was with the Department of Applied Physics, California Institute of Technology, Pasadena, CA 91125 . He is now deceased.

D. Mehuys and A. Yariv are with the Department of Applied Physics, California Institute of Technology, Pasadena, CA 91125.

IEEE Log Number 8714529 . cause the highest order supermode to have the highest modal gain, and this mode has a twin-lobed far-field pattern [5], [6]. In principle, one method of achieving fundamental mode operation in an array would be to vary (e.g., chirp) the widths of the array elements so that the fundamental mode is localized in a different spatial region than the higher order modes [7]. If the gain profile across the array is tailored to match the intensity distribution of the fundamental supermode, the result should then be an array with a single-lobed far-field pattern.

Unfortunately, fundamental physical and technological limitations make the fabrication of a real index-guided chirped array difficult [8], and there is as of yet no easy way to simultaneously control both the real index and gain distribution within a semiconductor laser. However, by taking advantage of the fact that in a gain-guided laser the spatial gain profile determines both the optical field distributions as well the modal gains, we demonstrated a tailored gain chirped array of semiconductor lasers in which gain tailoring was achieved by chirping the widths of the proton implanted laser elements [9]. Subsequently, we showed that the desired single-lobed far-field patterns could best be obtained from devices in which the interchannel gain has been made so strong that the distinction between an array and a broad area laser has become blurred [10].

The importance of a high interchannel gain in a tailored gain array has also recently been confirmed by the work of Welch and Scifres et al. [11], [12]. These workers also use proton implanted chirped arrays to provide gain tailoring, but rather than use a shallow proton implantation to achieve the necessary high interchannel gain, they utilize an offset stripe structure which effectively creates gain in the interchannel region. They have shown that singlelobed far-field patterns are best achieved when the length of the center section of the array equals the length of the two offset end sections. This implies that there is effectively no distinction between the channel and interchannel regions, because the total integrated gain along the length of the laser is (approximately) the same for any longitudinal cross section. Thus, the offset stripe "array" also resembles a tailored gain broad area laser more than it does an array of individual lasers.

\section{Tailored Gain Broad Area Lasers}

It is possible that some residual effect due to the array structure was responsible for the single-lobed far-field 
(a)

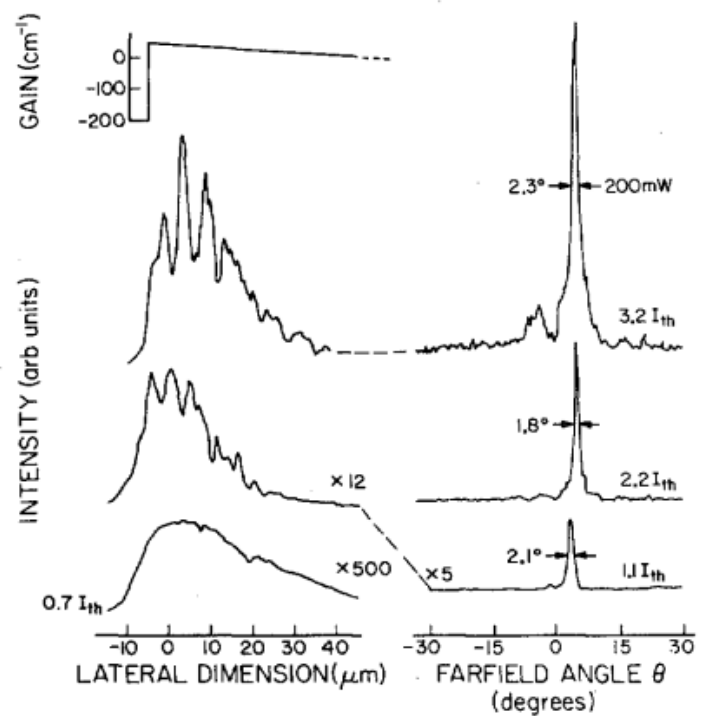

Fig. 1. Experimental (a) near-field and (b) far-field patterns for a halftone laser $100 \mu \mathrm{m}$ wide. Note the nearly linear spatial gain profile as evidenced by the spontaneous emission pattern at $0.7 I_{\mathrm{th}}$.

patterns. To demonstrate that the array structure in a tailored gain laser is superfluous, we therefore conceived and demonstrated an entirely different method of achieving gain tailoring, the "halftone" process, by varying the fractional coverage of injecting metal to the $\mathrm{p}^{+} \mathrm{GaAs}$ contact relative to Schottky blocking metal to the pGaAlAs contact over the surface of a broad area laser [13]. The enhanced current spreading provided by the thick upper cladding layer smears out the effects of the discrete dots and makes for a smooth, nonuniform spatial gain distribution within the active layer. This process may be used to create nearly arbitrary two dimensional spatial gain distributions within a broad area laser, thus offering a new degree of freedom in the design of semiconductor lasers. In particular, if the gain dependence on injected carrier density is known, the dot size variation can be chosen to create an approximately linear spatial gain profile. Previously, we were able to obtain a single-lobed diffraction limited beam from a tailored gain broad area laser $40 \mu \mathrm{m}$ wide [13]. The far-field patterns of this device did not remain single-lobed at high power; in this work, we report high-power $(200 \mathrm{~mW})$ nearly diffraction limited singlelobed operation of a similar device.

Fig. 1 shows the pulsed, low duty cycle near-field and far-field patterns for a linear asymmetric halftone laser $100 \mu \mathrm{m}$ wide. Fig. 1(b) shows that such lasers are capable of nearly diffraction limited high-power $(\sim 200 \mathrm{~mW}$ into $2.5^{\circ}$ ) single-lobed operation. The improved high-power performance may be understood as a consequence of the effect of gain saturation, which tends to equalize the spatial gain profile across the laser. The beneficial effects of gain tailoring are then lost more rapidly in a device with a small gain gradient (previous work) when compared with a device with a steeper gain gradient (present work).

The threshold currents $I_{\text {th }}$ and external differential quantum efficiencies $\eta_{\text {ext }}$ of tailored gain lasers depend upon (a)

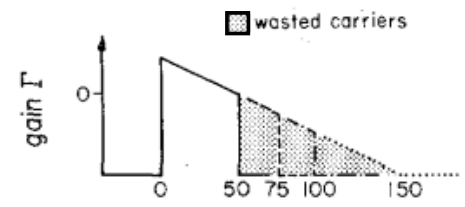

(b)

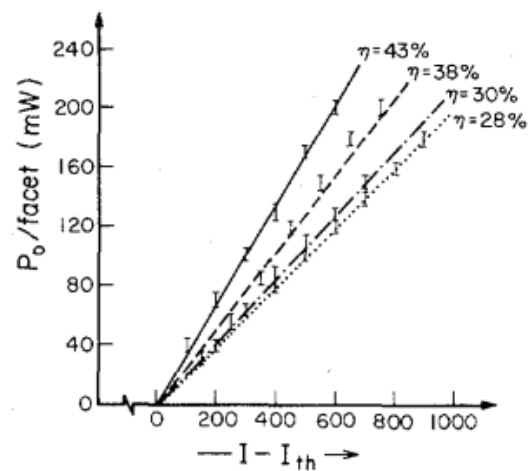

Fig. 2. Light-current curves for tailored gain broad area lasers. (a) Waveguide model for various truncated waveguides. The shaded areas represent regions of the waveguide which are pumped below transparency, and therefore waste carriers. (b) Experimental light-current curves showing variation of two mirror differential quantum efficiency $\eta$ as a function of device width.

the extent to which the waveguide is truncated at the low gain region because light is only emitted over those portions of the laser which are pumped to transparency $(\geq 0$ $\mathrm{cm}^{-1}$ ). Thus, carriers injected into the net lossy regions of the laser [indicated by the shaded regions of Fig. 2(a)] will increase $I_{\text {th }}$ and decrease $\eta_{\text {ext }}$ but will not increase the optical output. Fig. 2(b) plots the excess current above threshold $I-I_{\text {th }}$ versus the optical power emitted per facet for pulsed, low duty cycle operation of lasers with uncoated mirrors and for various device widths $\ell$. The total (two mirror) differential quantum efficiency $\eta_{\text {ext }}$ is also indicated. As expected, $\eta_{\text {ext }}$ rises as the width of the laser decreases. However, decreasing the width of the laser makes it more susceptible to the adverse effects of gain saturation, leading to some power being radiated into a small sidelobe at $-\theta$.

In this work, we restrict our attention primarily to the analytic study of the (unsaturated) optical eigenmodes of a linear asymmetric tailored gain waveguide. We will show that the highly nonuniform gain profile in halftone tailored gain broad area lasers plays an important role in the suppression of the lateral mode control problem in these devices. For example, we show that the higher order lateral modes of these lasers have far-field patterns which are all single-lobed and only slightly displaced from the fundamental. Thus, even under multilateral mode operation, the far-field pattern remains single-lobed, albeit with a slightly larger beamwidth.

In addition, the beam emission angle at threshold is sensitive to the exact value of the anti-guiding parameter [14], and we are therefore able to make use of asymmetric halftone tailored gain broad area lasers with varying spatial gain gradients to make a measurement of this important parameter. 


\section{Analysis of the Linear Tailored Gain WAVEGUIDE}

The optical field $\mathbf{E}(\mathbf{r}, t)$ inside the waveguide satisfies Maxwell's wave equation

$$
\nabla^{2} \mathbf{E}-\frac{n^{2}(\mathbf{r})}{c^{2}} \frac{\partial^{2} \mathbf{E}}{\partial t^{2}}=0
$$

where $c$ is the speed of light in vacuum, and $n(\mathbf{r})$ is the index of refraction in the medium, which is, in general, a complex number:

$$
\begin{aligned}
n(\mathbf{r}) & =\bar{n}(\mathbf{r})+i \overline{\bar{n}}(\mathbf{r}) \\
& =\bar{n}(\mathbf{r})-i \Gamma(\mathbf{r}) / 2 k_{0} .
\end{aligned}
$$

and $i=\sqrt{-1}$. (Throughout this work, the real part of a complex quantity $q$ will be denoted by either $\bar{q}$ or $\operatorname{Re}\{q\}$; the imaginary part will be denoted by either $\overline{\bar{q}}$ or $\operatorname{Im}\{q\}$ ). Hence, $\bar{n}(\mathbf{r})$ denotes the ordinary (real) index of refraction, and $\Gamma(\mathbf{r})=-2 k_{0} \overline{\bar{n}}(\mathbf{r})$ is the spatially dependent power gain experienced by an optical wave propagating through the point $\mathbf{r}$.

In a semiconductor laser, $\mathbf{E}(\mathbf{r}, t)$ is a complicated superposition of many transverse, lateral, and longitudinal modes oscillating at many different frequencies. We simplify the problem by considering only one oscillation frequency (thus eliminating the longitudinal modes), and make the usual effective index approximation (thereby eliminating the transverse modes). Furthermore, we consider only TE waves traveling in the $+z$ direction. As a result, we write the electric field of a lateral mode as

$$
\mathbf{E}(\mathbf{r}, t) \simeq \hat{\mathbf{x}} E(x) e^{i(\beta z-\Omega t)}, \beta=k_{0} \eta
$$

where $E(x)$ is now a scalar electric field, $k_{0}=2 \pi / \lambda_{0}$ is the free-space propagation constant, $\Omega=c k_{0}$ is the radian frequency of the wave, and $\eta$ is the effective index of the mode. The propagation constant $\bar{\beta}$ in the $z$ direction is then given by $\bar{\beta}=k_{0} \overline{\bar{\eta}}$. The power modal gain, defined as $\gamma=-2 k_{0} \overline{\bar{\eta}}=-2 \overline{\bar{\beta}}$, gives the rate at which the intensity of a lateral mode grows with $z$. In a laser, the lateral mode with the highest modal gain will be the lasing mode at threshold.

Substituting (3) into (1) yields the scalar Helmholtz equation:

$$
\frac{d^{2}}{d x^{2}} E+k_{0}^{2}\left(n^{2}(x)-\eta^{2}\right) E=0 .
$$

In the linear asymmetric waveguide, $n(x)$ is given by

$$
n(x)= \begin{cases}n_{e} & -\infty<x \leq 0^{-} \\ n_{0}-\sigma k_{0} x & 0^{+} \leq x \leq \ell^{-} \\ n_{e} & \ell^{+} \leq x<\infty\end{cases}
$$

where $n_{e}$ is the (constant) complex index of refraction external to the guide, $n_{0}$ and $\sigma$ are also complex constants, and $\ell$ is the width of the guide. For convenience, we split $n_{e}$ and $n_{0}$ into their real and imaginary parts:

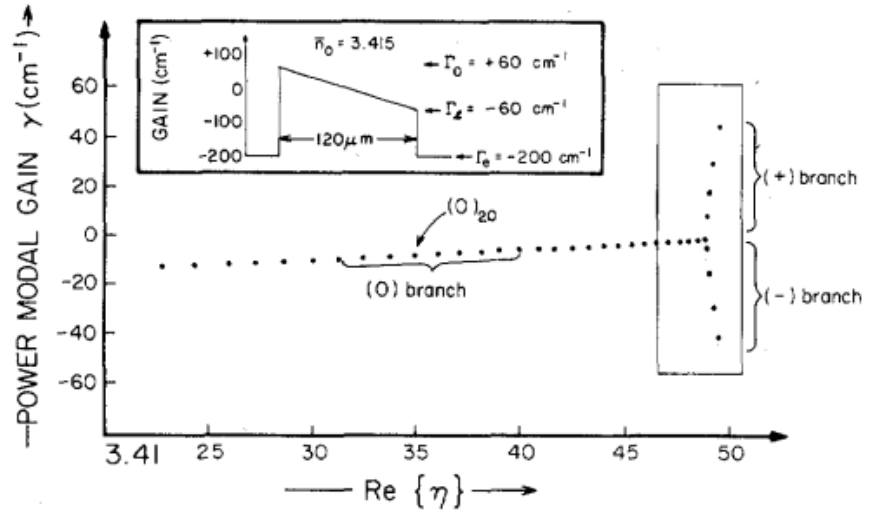

(a)

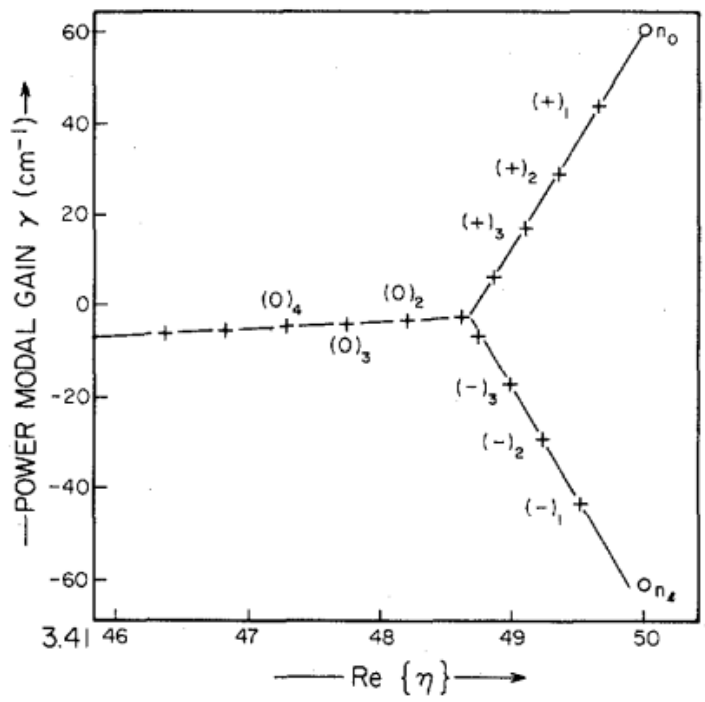

(b)

Fig. 3. (a) Mode diagram for an asymmetric linear tailored gain waveguide showing location of eigenmodes in the complex $\eta$ plane. (b) Enlargement of boxed region in part (b) showing modes on the $(+),(0)$, and $(-)$ branches.

$$
\begin{aligned}
& n_{e}=\bar{n}_{e}-i \Gamma_{e} / 2 k_{0} \\
& n_{0}=\bar{n}_{0}-i \Gamma_{0} / 2 k_{0} .
\end{aligned}
$$

The inset of Fig. 3(a) shows the waveguide which we will use for illustrative purposes throughout this work. It is $120 \mu \mathrm{m}$ wide with a gain gradient of $1 \mathrm{~cm}^{-1} / \mu \mathrm{m}$.

The gradient of the complex index of refraction within the guide $0 \leq x \leq \ell$ is given by $\sigma k_{0} . \sigma$ is a dimensionless quantity which plays a key role in determining the properties of the waveguide, and is defined by

$$
\sigma=-s(b+i) \quad(s, b>0)
$$

where $b$ is the antiguiding factor which relates the depression in the real part of the index of refraction due to the presence of gain through the free-carrier and band-edge effects. $b$ has been assigned values in the literature [14] between 2 and 6; as described below, we experimentally measure $b=2.5 \pm 0.5$. The real constant $s$ is related to the guide parameters by

$$
s=\frac{\Gamma_{0}-\Gamma_{\ell}}{2 k_{0}^{2} \ell} .
$$


It should be noted that in this model there will always be a step discontinuity in the index of refraction $n(x)$ at the left edge of the guide $(x=0)$, while there will be a corresponding step discontinuity at the right edge of the guide $(x=\ell)$ only if $\Gamma_{\ell} \neq \Gamma_{e}$ (i.e., $n_{e} \neq n_{\ell}=n_{0}-\sigma k_{0} \ell$ ). The effect of the discontinuity in $n(x)$ at the right edge of the guide depends upon the magnitude of the discontinuity, and also upon both the particular eigenmode as well as the width $\ell$ of the guide. Of course, in an actual device there can be no real discontinunity in the spatial gain profile, but since we will show below that it is only the value of the spatial gain gradient which is important, to first order at least, the discrepancy between the model waveguide and an actual device may be neglected for the high gain lasing modes.

After substituting (5) into (4) and dropping the term second order in $x$, the Helmholtz equation inside the guide becomes

$$
\frac{d^{2}}{d x^{2}} E+k_{0}^{2}\left(\left(n_{0}^{2}-\eta^{2}\right)-2 n_{0} k_{0} \sigma x\right) E=0
$$

which has the solution

$$
E(x)=a \operatorname{Ai}(z)+b \operatorname{Bi}(z), \quad z=\rho+\omega x
$$

where $a$ and $b$ are complex constants, $\operatorname{Ai}(z)$ and $\operatorname{Bi}(z)$ are the Airy functions, and

$$
\begin{aligned}
& \rho=\frac{k_{0}^{2}}{\omega^{2}}\left(\eta^{2}-n_{0}^{2}\right) \\
& \omega=k_{0}\left(2 n_{0} \sigma\right)^{1 / 3} .
\end{aligned}
$$

The equation for the argument to the Airy functions, $z=$ $\rho+\omega x$, describes a straight line $\mathfrak{L}$ in the complex $z$-plane with one endpoint at $z=\rho$ and the other endpoint at $z=$ $\rho+\omega \ell$. The length of this line is $|\omega \ell|$, while the angle $\psi$ the line makes with the real axis is given by $\psi=\angle \omega=$ $\tan ^{-1} \operatorname{Im}\{\omega\} / \operatorname{Re}\{\omega\}$. For the case of pure index guiding, $\psi$ is zero, and $\mathscr{L}$ lies on the real axis. In the case of pure gain guiding $(b=0)$, as considered in Fig. 3, $\sigma$ is a pure imaginary number:

$$
\sigma=-i s \quad \text { (pure gain guiding, } s \text { real) }
$$

where $s$ is related to the guide parameters by (8). Then we can write $\omega$ as

$$
\omega=k_{0}\left(2 n_{0} s\right)^{1 / 3}(-i)^{1 / 3} \text {. }
$$

Each branch of the cube root gives rise to a physically meaningful mode, leading to three distinct families of modes, which will be referred to as the $(+),(0)$, and ( - ) branches. Since $\overline{\bar{n}}_{0}<<\bar{n}_{0}$, the angle $\psi$ is determined almost entirely by the cube root of $(-i)$ which takes the values $e^{-i \pi / 6}, e^{-i 5 \pi / 6}$, and $e^{+i \pi / 2} \cdot \psi$ then takes on the values $\mp 30^{\circ}$, and $+90^{\circ}$. The inclusion of index anti-guiding $(b \neq 0)$ effects a rotation of $\mathscr{L}$ in the complex plane; this will be discussed below.

The quantity $\omega l$ determines the length and orientation of the line $\mathscr{L}$ in the complex plane. To determine its origin at $\rho$ the eigenvalue $\eta$ is required. The normalized eigen- modes will then be completely specified when the ratio of the coefficients $a / b$ in (10) is known. This ratio is determined by requiring $E$ and $d E / d x$ to be continuous at the edges of the guide and bounded at infinity. If we require the field to decay exponentially as $x \rightarrow-\infty$ and match the boundary conditions at the left edge of the guide, we derive the following expression for the ratio $a / b$ inside the guide:

$$
\left.\frac{a}{b}\right|_{x=0}=-\frac{\sqrt{\rho_{e}} \mathrm{Bi}(\rho)-\mathrm{Bi}^{\prime}(\rho)}{\sqrt{\rho_{e}} \operatorname{Ai}(\rho)-\mathrm{Ai}^{\prime}(\rho)}
$$

where the prime $\left({ }^{\prime}\right)$ denotes a derivative with respect to the argument, and

$$
\rho_{e}=\frac{k_{0}^{2}}{\omega^{2}}\left(\eta^{2}-n_{e}^{2}\right)
$$

Similar consideration at the right-hand side of the guide leads to

$$
\left.\frac{a}{b}\right|_{x=\ell}=-\frac{\sqrt{\rho_{e}} \operatorname{Bi}(\rho+\omega \ell)+\mathrm{Bi}^{\prime}(\rho+\omega \ell)}{\sqrt{\rho_{e}} \operatorname{Ai}(\rho+\omega \ell)+\mathrm{Ai}^{\prime}(\rho+\omega \ell)} .
$$

Inside the guide, the ratios $\left.(a / b)\right|_{x=0}$ and $\left.(a / b)\right|_{x=\ell}$ both describe the same linear combination of $\mathrm{Ai}(z)$ and $\operatorname{Bi}(z)$. Setting them equal yields the eigenvalue equation for $\eta$ :

$$
\begin{aligned}
& \frac{\sqrt{\rho_{e}} \operatorname{Bi}(\rho)-\mathrm{Bi}^{\prime}(\rho)}{\sqrt{\rho_{e}} \operatorname{Ai}(\rho)-\operatorname{Ai}^{\prime}(\rho)} \\
& \quad-\frac{\sqrt{\rho_{e}} \operatorname{Bi}(\rho+\omega \ell)+\operatorname{Bi}^{\prime}(\rho+\omega \ell)}{\sqrt{\rho_{e}} \operatorname{Ai}(\rho+\omega \ell)+\operatorname{Ai}^{\prime}(\rho+\omega \ell)}=0 .
\end{aligned}
$$

This equation may also be obtained by the more common method of solving a $4 \times 4$ determinant; the present method has the advantage that it explicitly yields the important ratio $a / b$.

Equation (17) is solved numerically for the eigenvalues $\eta_{\nu}$, where $\nu$ is the mode index. The mode structure in the complex effective index plane for our model waveguide is illustrated in Fig. 3(a). An enlargement of the boxed region of Fig. 3(a) is shown in Fig. 3(b). Three distinct families of modes exist in a pattern resembling a sideways " $Y$ "': the high modal gain $(+)$ and the low modal gain $(-)$ branches with relatively large $\bar{\eta}$, and the middle (0) branch on which all the modes have nearly the same modal gain and relatively small $\bar{\eta}$. Unlike the case of a uniform gain guide of the same width in which the mode discriminations are less than $1 \mathrm{~cm}^{-1}$, the mode discrimination between the fundamental and higher order modes in an asymmetric tailored gain waveguide can be much better, typically $10 \mathrm{~cm}^{-1}$. Since the lateral modes on the $(+)$ branch will be the first to lase, these are then the most important ones to characterize. To accomplish this, we examine the Airy functions of complex argument.

It is well known that along the negative real axis, $\mathrm{Ai}(x)$ resembles a damped sinusoidal function with a gradually decreasing period, while along the positive real axis $\mathrm{Ai}(x)$ 
$|A i(z)|$

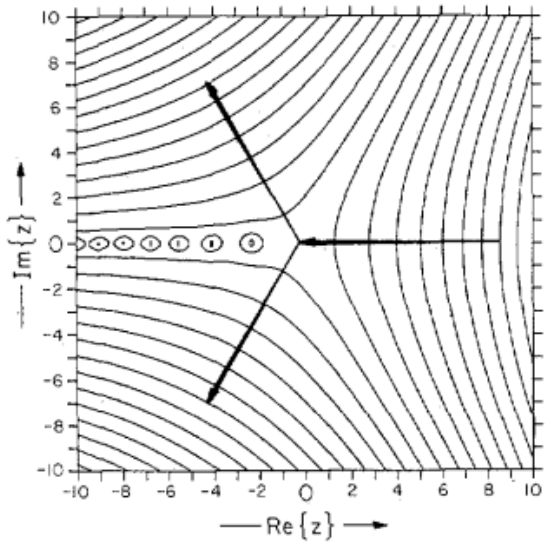

(a)

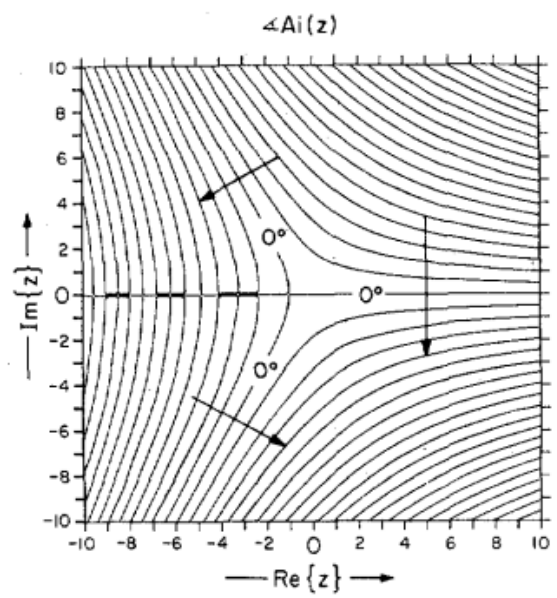

(c)

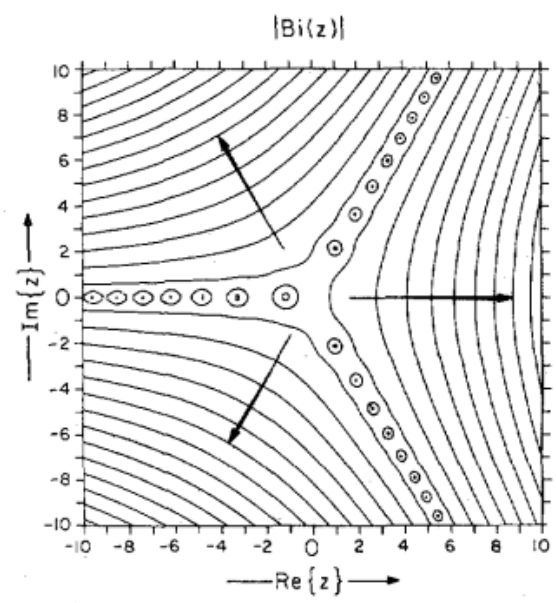

(b)

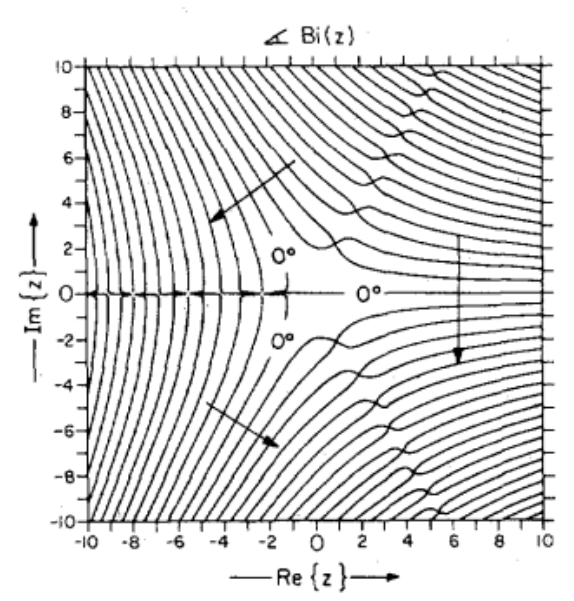

(d)

Fig. 4. Level lines for the magnitude of (a) $\mathrm{Ai}(z)$ and (b) $\mathrm{Bi}(z)$; the arrows show the direction of increasing magnitude. Lines of constant phase for (c) $\mathrm{Ai}(z)$ and (d) $\mathrm{Bi}(z)$; the arrows show the direction of increasing phase. The contours in (a) and (b) differ by a factor of ten and by $\pi / 4$ in (c) and (d).

decays exponentially without oscillations or zeros. Similarly, along the negative real axis, $\mathrm{Bi}(x)$ resembles a damped cosinusoidal function with a gradually decreasing period, while along the positive real axis $\mathrm{Bi}(x)$ grows exponentially without oscillations or zeros. However as gain is introduced into the waveguide, the mode paths deviate from the real axis and the eigenmodes of this complex waveguide are determined by the analytic continuations of $\mathrm{Ai}(x)$ and $\mathrm{Bi}(x)$ into the complex plane. For convenience, the magnitude and phase of both Airy functions are illustrated over the complex plane in Fig. 4. The arrows indicate directions of increasing magnitude and phase.

In order to proceed with the theoretical analysis, it is necessary to determine which one of the terms $a \mathrm{Ai}(x)$ or $b \operatorname{Bi}(z)$ provides the dominant contribution to $E$ in (10). Towards that end, we examine the paths $\mathscr{L}(+), \mathfrak{L}(0)$ and $\mathscr{L}(-)$ of the argument $z=\rho+\omega x$. For example, the paths $\mathscr{L}(+)$ are shown superimposed on $\operatorname{Ai}(z)$ in Fig. 5(a). The endpoint at $z=\rho$ lies near a zero of $\operatorname{Ai}(z)$ and the other is in the sector $|\angle z|<\pi / 3$ where $\operatorname{Ai}(z)$ is exponentially small. Thus the profile of $\operatorname{Ai}(z)$ along this path is consistent with a well-confined mode. From Fig. 4 we note that in the sector $|\angle z|<\pi / 3, \mathrm{Bi}(z)$ is exponentially large, so that its magnitude at $z=\rho+\omega \ell$ is much larger than it is at $z=\rho$. Its profile is not consistent with a well-confined mode and therefore we expect the ratio $a / b$ to be large for modes on the $(+)$ branch. This ratio is plotted in Fig. 6 for a guide with a gain gradient of $1 \mathrm{~cm}^{-1} / \mu \mathrm{m}$ and varying widths $\ell$. The ratio $a / b$ approaches values as high as $10^{12}$, confirming that the contribution of $\mathrm{Ai}(z)$ to $E$ completely dominates that of $\mathrm{Bi}(z)$ for the first few modes of guides wider than about $50 \mu \mathrm{m}$. As the guide width $\ell$ is increased, the endpoint $z=\rho+$ $\omega \ell$ penetrates deeper into the Stokes region $|\angle z|<\pi / 3$ and the increasing ratio $a / b$ reflects the growing dissimilarity between $\mathrm{Ai}(z)$ and $\operatorname{Bi}(z)$. Only when the endpoint $z=\rho+\omega \ell$ lies near the Stokes line at $\angle z=\pi / 3$ does $\operatorname{Bi}(z)$ make a significant contribution to the eigenmode. Thus the solution $E_{v}^{(+)}(x)$ of the Helmholtz equation (4) can be expressed in terms of $\mathrm{Ai}(z)$ alone in guides for which $|\omega| \ell$ is suitably large.

The near-field profiles along lines $\mathscr{L}(+)$ are shown in Fig. 7(a). These are virtually identical to those obtained 


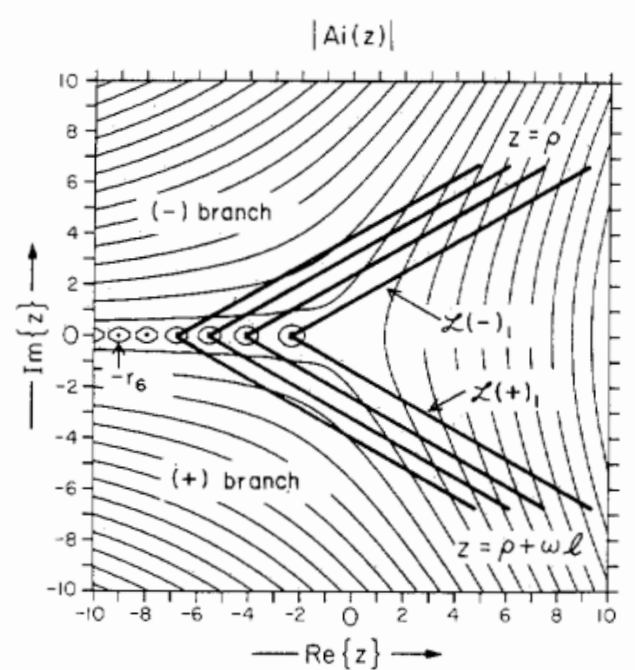

(a)

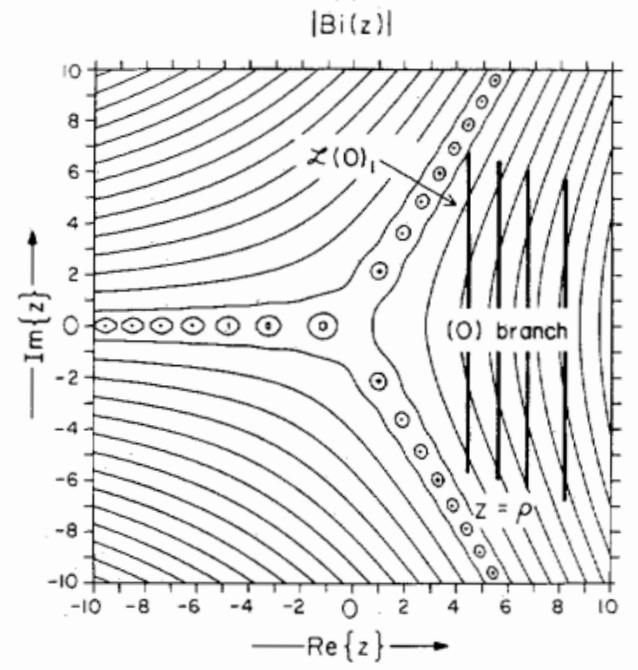

(b)

Fig. 5. Path of the argument to the Airy function for the waveguide of Fig. 3(a). (a) $(+)$ and $(-)$ branches. (b) (0) branch.

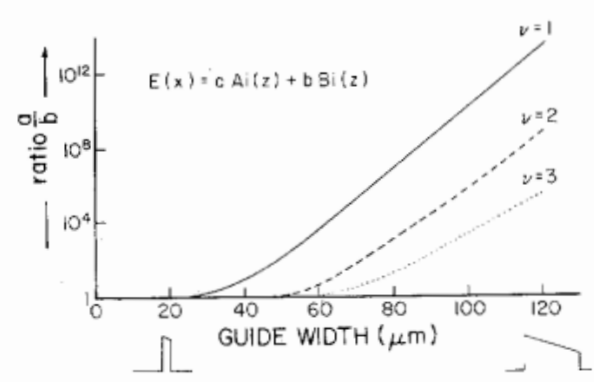

Fig. 6. Ratio of $\operatorname{Ai}(z) / \operatorname{Bi}(z)$ for the first three modes on the $(+)$ branch for guides with a gain gradient $1 \mathrm{~cm}^{-1} / \mu \mathrm{m}$ and different widths $\ell$. To an excellent degree of approximation, low order modes on the $(+)$ branch are determined almost entirely by the behavior of $\mathrm{Ai}(z)$.

by a completely numerical study of the problem [8]. Note that these modes are concentrated near the high-gain end of the waveguide $(x=0)$. The $\nu=1$ mode has the largest spatial overlap with the lateral gain profile and thus has the highest modal gain at threshold. As is evident from Fig. 3(b) it also has the highest effective index of any mode on the $(+)$ branch, and will henceforth be referred to as the "fundamental" mode of the waveguide.

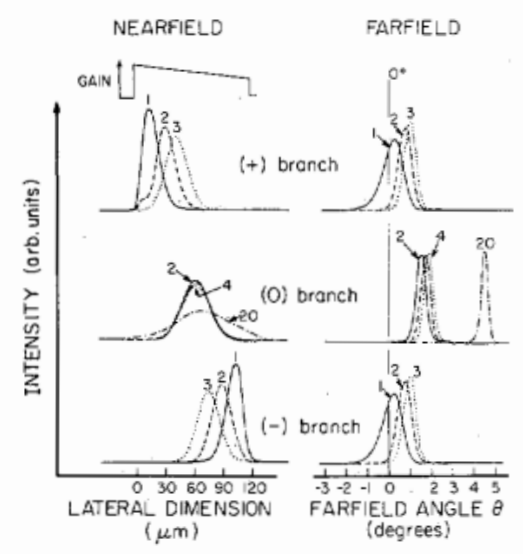

(a)

(b)

Fig. 7. (a) Intensity near-field patterns and (b) far-field patterns for the modes of Fig. 3.

Similar arguments can be applied to both the $(0)$ and (-) branches. The paths $\mathscr{L}(-)$ with $\psi=+30^{\circ}$ are also illustrated in Fig. 5(a), and are similar to $\mathscr{L}(+)$ in that the $\operatorname{Ai}(z)$ function is best suited to describe the mode. Since here the zero of the Airy function occurs at $z=\rho$ $+\omega \ell$, the near-fields are concentrated in the lossy regions of the waveguide, as illustrated in Fig. 7(a).

The path of the argument of the Airy functions for the (0) branch is plotted in Fig. 5(b) with $\psi=+90^{\circ}$. Unlike the previous two cases, here $\operatorname{Bi}(z)$ provides the best description of a confined mode. Due to the symmetry of $\operatorname{Bi}(z)$ about the positive real axis the near-field patterns of these modes are approximately centered within the waveguide. These modes therefore have a modal gain between modes on the $(+)$ branch and modes on the $(-)$ branch. They correspond to the (0) branch of Fig. 3, and are also plotted in Fig. 7. We remark that this description of modes on the $(0)$ branch holds for the wide waveguides considered here, but becomes considerably more complicated for waveguides in which $|\omega \ell| \rightarrow 0$.

\section{Eigenvalues of the Linear Tailored Gain W AVEGUIDE}

Once it has been determined that the contribution of $\mathrm{Ai}(z)$ dominates that due to $\operatorname{Bi}(z)$ on the principal $(+)$ branch, it becomes possible to derive very simple analytical expressions for the mode effective indexes and modal gains by recalling that $\mathscr{L}(+)$ starts near a zero of $\mathrm{Ai}(x)$ and ends in the sector of exponential decay. At the left edge of the guide $E(0)=a \operatorname{Ai}(\rho)$, where $\rho$ is given by (11). Setting $\rho$ equal to one of the (real) zeros $-r_{\nu}$ of the Airy function gives

$$
-r_{p} \simeq \rho_{v}=\frac{k_{0}^{2}}{\omega^{2}}\left(\eta_{\nu}^{2}-n_{0}^{2}\right)
$$

and approximating $\left(\eta_{v}^{2}-n_{0}^{2}\right)$ by $2 n_{0}\left(\eta_{v}-n_{0}\right)$ yields an expression for the $\nu^{\text {th }}$ eigenvalue $\eta_{v}$ :

$$
\eta_{v} \simeq n_{0}-\frac{1}{2 n_{0}} \frac{\omega^{2}}{k_{0}^{2}} r_{\nu}
$$


The $\nu^{\text {th }}$ zero of $\mathrm{Ai}(z)$ is approximately [15]

$$
-r_{\nu} \simeq\left[\frac{3}{2}\left(\nu-\frac{1}{4}\right) \pi\right]^{2 / 3} .
$$

After using the definition of $\omega$ in (11) for the case of pure gain guiding ( $\sigma=-i s$ ), taking the principal branch of $\omega$, and equating the real and imaginary parts we obtain an expression for $\eta=\bar{\eta}+i \overline{\bar{\eta}}$ on the $(+)$ branch:

$$
\begin{aligned}
& \bar{\eta}_{\nu}^{(+)} \simeq \bar{n}_{0}-\frac{1}{2} \epsilon_{\nu} \\
& \overline{\bar{\eta}}_{\nu}^{(+)} \simeq \overline{\bar{n}}_{0}+\frac{\sqrt{3}}{2} \epsilon_{\nu}
\end{aligned}
$$

where

$$
\epsilon_{\nu}=\left|\frac{s^{2}}{2 n_{0}}\right|^{1 / 3} r_{\nu} .
$$

In the complex $\eta$ plane, therefore, the mode structure is particularly simple: all the modes of the principal branch lie on a straight line emanating from the point $\left(\bar{n}_{0}, \overline{\bar{n}}_{0}\right)$ and making an angle $\tan ^{-1}(-\sqrt{3})=-60^{\circ}$ with the real axis. The modes are spaced along this line according to the zeros of the Airy function, with the higher order modes being more closely spaced together.

Since the modal gain $\gamma_{v}^{(+)}$is related to $\overline{\bar{\eta}}_{v}$ through (2),

$$
\begin{aligned}
\gamma_{\nu}^{(+)} & \simeq \Gamma_{0}-k_{0} \sqrt{3} \epsilon_{\nu} \\
& \simeq \Gamma_{0}-\sqrt{3} k_{0}\left|\frac{s^{2}}{2 n_{0}}\right|^{1 / 3} r_{\nu}
\end{aligned}
$$

and $0<r_{1}<r_{2} \cdots$, we see that the fundamental mode has the highest modal gain and hence will be the lasing mode at threshold. At threshold, the modal gain $\gamma_{1}^{(+)}$of the fundamental mode must equal the mirror losses $-\Gamma_{m}$ (scattering losses are probably insignificant in such a wide gain-guided laser). Equation (23) may be inverted to give the required peak gain $\Gamma_{0}$ at threshold in terms of $\Gamma_{m}$ and the gain gradient $s$.

The mode discrimination between any two modes is given by

$$
\begin{aligned}
\Delta \gamma_{v}^{(+)} & \equiv \gamma_{\nu}^{(+)}-\gamma_{\nu+1}^{(+)} \\
& \simeq \sqrt{3} k_{0}\left|\frac{s^{2}}{2 n_{0}}\right|^{1 / 3} \delta_{v} \delta_{v} \equiv\left|r_{v}-r_{\nu+1}\right|
\end{aligned}
$$

where $\delta_{y}$ is the spacing between the zeros of $\operatorname{Ai}(x)$. The mode discrimination scales sublinearly with the gain gradient, and is greatest between the fundamental $\nu=1$ and the next higher-order $\nu=2$ mode. Our numerical analysis [8] indicates that (24) is accurate to within a few percent for the waveguide of Fig. 3(a).

In an entirely analogous manner, formulas for the propagation constants of modes on the $(-)$ branch may be determined by setting $\rho+\omega \ell$ equal to a zero of the Airy function. The effect of the additional term $\omega \ell$ and the $(-)$ root of $\omega$ leads to slightly different expressions for the eigenvalues $\eta_{v}^{(-)}$and modal gain $\gamma_{v}^{(-)}$:

$$
\begin{aligned}
& \bar{\eta}_{\nu}^{(-)} \simeq \bar{n}_{\ell}-\frac{1}{2} \epsilon_{\nu} \\
& \overline{\bar{\eta}}_{\nu}^{(-)} \simeq \overline{\bar{n}}_{\ell}-\frac{\sqrt{3}}{2} \epsilon_{\nu} \\
& \gamma_{\nu}^{(-)} \simeq \Gamma_{\ell}+\sqrt{3} k_{0}\left|\frac{s^{2}}{2 n_{0}}\right|^{1 / 3} r_{\nu} .
\end{aligned}
$$

The approximations for the eigenvalues on the $(-)$ branch lie on a straight line emanating from the point $\left(\bar{n}_{\ell}, \overline{\bar{n}}_{\ell}\right)$. The angle that this line makes with the real axis is $+60^{\circ}$, so that it makes an angle of $120^{\circ}$ with the corresponding (+) line. In this case the $\nu=1$ mode on the (-) branch has the lowest modal gain of all the modes. We remark that (25) is not as accurate as (23) for truncated waveguides in which $n_{\ell} \neq n_{0}-\sigma k_{0} \ell$ due to the perturbation introduced by the discontinuous truncated region at the lossy edge of the guide. As a result of their relatively low modal gain, modes on the (-) branch are unlikely to lase, however, and so the error is unimportant from a practical point of view.

Finally, modes on the $(0)$ branch have nearly constant modal gains, and are composed almost entirely of $\operatorname{Bi}(z)$. It is not possible to obtain simple closed form analytic expressions for the eigenvalues for these modes. Once again, however, since they have low modal gains compared to those on the $(+)$ branch, the formulas are not required.

\section{Near-Field and Far-Field Patterns}

The near-field patterns of the lowest order eigenmodes of our model waveguide were illustrated in Fig. 7. Considering first the $(+)$ branch, we recall that $\mathfrak{L}$ starts from a zero of $\mathrm{Ai}(z)$ and terminates in the sector of exponential decay $|<| z<\pi / 3$. Since the zeros of $\operatorname{Ai}(z)$ occur only along the negative real axis, the modes of an asymmetric linear tailored gain waveguide all have single-lobed nearfield patterns.

Prior to deriving analytic approximations for the nearfields, we determine the position within the guide, $\chi_{\nu}$, of peak intensity of the $\nu^{\text {th }}$ mode. Referring to Fig. 8, we see that along the path $\mathscr{L},|\mathrm{Ai}(z)|$ reaches its maximum when $\mathcal{L}$ is tangent to the level lines of $\operatorname{Ai}(z)$. These level lines are perpendicular to the lines of constant phase of $\mathrm{Ai}(z)$. The anti-Stokes line associated with the principal branch is asymptotic to [see Fig. 4(c)] the line of constant phase which makes an angle of $-60^{\circ}$ with the negative real axis. The corresponding tangent line is at an angle of $-30^{\circ}$, which is precisely the angle $\psi$ that $\mathscr{L}$ makes with the negative real axis for the special case of no index antiguiding. Setting $\rho_{\nu} \simeq-r_{\nu}$, a simple geometric construction yields the following expression for $\chi_{v}$ :

$$
\chi_{\nu}=\frac{\sqrt{3}}{2} \frac{r_{\nu}}{|\omega|} .
$$




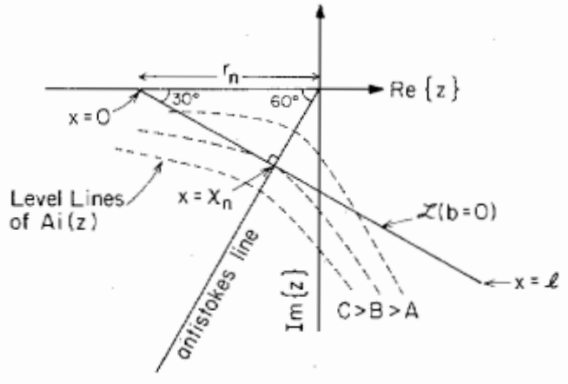

Fig. 8. Calculation of the near-field pattern showing geometric construction for determining $\chi_{\nu}$.

It should be noted that, for a wide guide, the position of the mode within the guide $\chi_{v}$ depends only on the gain gradient, and is independent of both the peak gain $\Gamma_{0}$ and the width $\ell$ of the guide. The mode maxima are separated within the guide by [using (11)]

$$
\Delta \chi_{v}=\frac{\sqrt{3}}{2 k_{0}}\left|2 n_{0} s\right|^{-1 / 3} \delta_{v}
$$

where $s$ is given by (8) and $\delta_{v}$ by (24).

Equation (27) leads to a particularly simple expression for the modal gains on the principal branch. Since $\Gamma(x)$ is the lateral gain profile and taking $g=\left(\Gamma_{0}-\Gamma_{\ell}\right) / \ell$ as the spatial gain gradient, the expressions for the modal gain and the mode discrimination become

$$
\begin{aligned}
\gamma_{\nu}^{(+)} & =\Gamma\left(\chi_{\nu}\right) \\
\Delta \gamma_{\nu}^{(+)} & =g \Delta \chi_{\nu} .
\end{aligned}
$$

That is, the modal gain is given simply by the value of the spatial gain at the point where the electric field has its peak value. This suggests that, to first order, the mode intensity profile is symmetric about its peak position, and that its width is much less than the width of the waveguide.

To find an expression for the near-field pattern, we approximate the Airy function $\mathrm{Ai}(z)$ along the lines $\mathscr{L}(+)$. To wit, the leading asymptotic behavior of $\mathrm{Ai}(z)$ as $|z|$ $\rightarrow \infty$ is [15] to the lateral position $x$ within the guide:

$$
\begin{aligned}
& u=\frac{r_{\nu}}{2} \\
& v=|\omega|\left(x-\chi_{\nu}\right) .
\end{aligned}
$$

Below the negative real axis, the + exponential provides the dominant contribution to $\mathrm{Ai}(z)$. The radicals may be simplified by making a binomial expansion about $v=0$ with $v<<u$ :

$$
\begin{aligned}
& (u+i v)^{3 / 2} \approx u^{3 / 2}\left[1+i \frac{3}{2} \frac{v}{u}-\frac{3}{8}\left(\frac{v}{u}\right)^{2}\right] \\
& (u+i v)^{-1 / 4} \approx u^{-1 / 4}\left[1+\left(\frac{v}{4 u}\right)^{2}\right]^{1 / 2} e^{-i(v / 4 u)} .
\end{aligned}
$$

After substituting (31) and (30) into (29) and dropping an unimportant constant, the expression for the electric field reduces to

$$
E(x) \simeq e^{-\left(x-\chi_{v}\right)^{2} / 2 \omega_{v}^{2}} e^{+i \phi_{\nu}\left(x-\chi_{\nu}\right)} .
$$

That is, the near-field pattern is approximately a Gaussian with half width $w_{\nu}$, centered at $\chi_{\nu}$ and multiplied by a linear phase variation $\phi_{\nu}$, where $\chi_{\nu}$ is given by (26), and $w_{\nu}$ and $\phi_{\nu}$ are given by

$$
\begin{aligned}
w_{\nu} & =\frac{\left(2 r_{\nu}\right)^{1 / 4}}{|\omega|} \\
\phi_{\nu} & =|\omega|\left[\left(\frac{r_{\nu}}{2}\right)^{1 / 2}-\frac{1}{2 r_{\nu}}\right] .
\end{aligned}
$$

The normalized near-field intensity $I_{\nu}(x)$ is therefore

$$
I_{\nu}(x) \simeq \frac{1}{\sqrt{\pi w_{\nu}^{2}}} e^{-\left(x-\chi_{\nu}\right)^{2} / w_{\nu}^{2}} .
$$

The intensity near-field patterns may be found for the $(-)$ branch in a similar manner. The exact (numerical) near-field intensities and phases for representative modes on each of the three branches is plotted in Fig. 9(a). All of the modes are approximately Gaussian in shape with essentially linear phase variations over the region of ap-

$$
\operatorname{Ai}(z) \sim \begin{cases}\frac{1}{2} \pi^{-1 / 2}(-z)^{-1 / 4}\left(e^{+i\left(2 / 3(-z)^{3 / 2}-\pi / 4\right)}+e^{-i\left(2 / 3(-z)^{3 / 2}-\pi / 4\right)}\right) & \frac{\pi}{3}<|<z|<\frac{5 \pi}{3} \\ \frac{1}{2} \pi^{-1 / 2} z^{-1 / 4} e^{-2 / 3 z^{3 / 2}} & |<z|<\pi\end{cases}
$$

The first expression includes the zeros on the negative real axis, and is the one we use. In constructing Fig. 4, we have found this result to be accurate to within 10 to 15 percent even for $|z|$ as small as 2 or 3 , and its simple analytical form further motivates its exploitation here.

We write $z$ in terms of the rectangular coordinates $z=$ $u^{\prime}+i v^{\prime}$ and rotate the coordinate system to new variables $u$ and $v$ so that $u$ lies along the anti-Stokes line $\angle z=$ $e^{-i 2 \pi / 3}$ and $v$ lies perpendicular to it (i.e., along $\mathscr{L}$ ). Along the line $\mathscr{L}, u$ is constant and $v$ is linearly related preciable light intensity. Higher order terms in the expansion of (31) lead to slightly asymmetric near-field patterns with some curvature in the phase fronts.

Once $E_{\nu}(x)$ has been found to have such a simple form, it is a simple matter to find its far-field pattern. In the Fraunhofer approximation, the far-field pattern $F_{\nu}(\theta)$ is given by the square of the Fourier transform of $E_{\nu}(x)$ times an obliquity factor. The wide asymmetric tailored gain broad area lasers of interest here have very narrow far-field patterns near the axis, and thus the obliquity fac- 
NEARFIELD

(a)

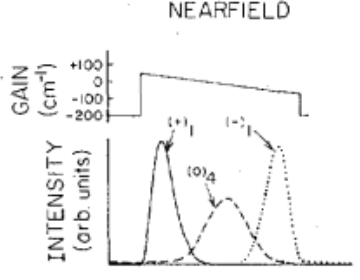

(c)

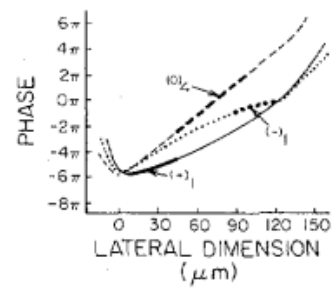

Fig. 9. (a) The waveguide of Fig. 3(a). (b) The near-field patterns of selected modes on each of the three branches. (c) The corresponding phases. Note that all the near-field patterns are approximately Gaussian in shape, and that the phase fronts are nearly linear.

tor may be ignored. Making use of the shift and convolution Fourier transform theorems, the intensity far-field pattern may be written as

$$
\begin{aligned}
F_{\nu}(\theta) & =\left|\mathcal{F}\left\{e^{-\left(x-\chi_{\nu}\right)^{2} / 2 \omega_{\nu}^{2}}\right\} \star \mathcal{F}\left\{e^{i \phi_{\nu}\left(x-\chi_{\nu}\right)}\right\}\right|^{2} \\
& =\left|e^{-\theta^{2} / 4 \Sigma_{v}^{2}} \star \delta\left(\theta-\theta_{\nu}\right)\right|^{2} \\
& =e^{-\left(\theta-\theta_{\nu}\right)^{2} / 2 \Sigma_{\nu}^{2}}
\end{aligned}
$$

where $\mathcal{F}\{\}$ denotes a $(-i)$ Fourier Transform, $\star$ denotes the convolution operation, and the emission angle $\Theta_{\nu}$ and beamwidth $\Sigma_{\nu}$ after refraction at the resonator facet are given by

$$
\begin{aligned}
& \Theta_{\nu}\left({ }^{\circ}\right)=\left(\frac{180}{\pi}\right) \frac{\phi_{\nu}}{k_{0}}=\left(\frac{180}{\pi}\right)|\omega|\left[\left(\frac{r_{\nu}}{2}\right)^{1 / 2}-\frac{1}{2 r_{\nu}}\right] \\
& \Sigma_{\nu}\left({ }^{\circ}\right)=\left(\frac{180}{\pi}\right) \frac{1}{k_{0}} \frac{1}{w_{\nu}}=\left(\frac{180}{\pi}\right) \frac{|\omega|}{k_{0}\left(2 r_{\nu}\right)^{1 / 4}} .
\end{aligned}
$$

Far-field patterns for the ( - ) branch may be calculated similarly, and are, in fact, identical (except for an unimportant global phase factor) to those on the $(+)$ branch.

The far-field patterns corresponding to all near-field modes of Fig. 7(a) are plotted in Fig. 7(b). We see that the far-field patterns for all modes are emitted slightly offaxis, are single-lobed and approximately Gaussian in shape, in agreement with (35). This stands in marked contrast to the far-field patterns of all real index-guided and symmetric gain-guided lasers in which only the fundamental mode has a single-lobed far-field pattern. Physically, we understand this as follows: all of the low-order eigenmodes are well confined within the guide. Hence each experiences a nearly linear gain gradient across its width. The self-consistent solution inside the laser resonator thus requires a net power flow from the side of high gain to the side of low gain. As a consequence the phase front of the guided wave is tilted with respect to the optical axis, and power is emitted in the far-field at an angle $\Theta_{\nu}$ off-axis towards the low gain side of the laser.
In real index-guided and symmetric gain-guided lasers, power flow directed off-axis at an angle $\theta_{\nu}$ must be balanced by an equal component at $-\Theta_{\nu}$. The asymmetric tailored gain waveguide disrupts this symmetry by reinforcing one component at the expense of the other. This may be seen analytically by examining (29). The + and - exponentials describe power propagation at opposite angles to the optical axis. Choosing the gain slope $s>0$ forces the lines $\mathscr{L}(+)$ below the negative real axis, and the + exponential is dominant (power flow reinforced at $\left.\Theta_{\nu}\right)$. On the other hand, choosing $s<0$ would cause the - exponential to dominate. On the negative real axis (Stokes line), neither term is dominant. This switching of dominance is exactly the Stokes phenomenon. Thus, a small amount of power which travels in the opposite lateral direction is described by the subdominant term of (29), which we neglected, and contributes to far-field emission at an angle $-\theta_{y}$.

We stress that our analysis is predicated on achieving distinct $(+)$ and $(-)$ branches in the mode structure. A suitable degree of asymmetry is required to accomplish this. Since the near-field patterns of the low-order eigenmodes on the $(+)[(-)]$ branch are strongly localized in the high gain (low gain) half of the guide, a number $\nu$ modes appears on each if the guide width $(\ell)$ and gain gradient (through $\omega$ ) satisfy

$$
\frac{|\omega| \ell}{2} \geq \chi_{\nu}+2 \frac{w_{\nu}}{|\omega|} \simeq \frac{\sqrt{3}}{2} r_{\nu}+2\left(2 r_{\nu}\right)^{1 / 4} \text {. }
$$

Of some practical importance is the fact that the farfield patterns of the higher order modes on the $(+)$ branch are only slightly displaced from the fundamental. From (20) and (36) we see that the angles of emission of higher order modes scale approximately as $\nu^{1 / 3}$. Thus, under high-power operation when multilateral mode operation is likely, the beamwidth will degrade gradually, becoming slightly broader and shifting very slightly in angle. This analytical result is borne out by the experimental data of Fig. 1(b).

\section{Vi. Measurement of the Antiguiding Parameter}

In the interest of simplicity, the preceding analysis considered only the case of a pure gain waveguide with no index variation. The effect of the antiguiding parameter $b$ on the eigenmodes may be determined by recalling the definition of $\sigma$ in (7), viz. $\sigma=-s(b+i)$. Rewriting $\sigma$ as

$$
\sigma(b)=\sigma(0)(1-i b)
$$

then

$$
\begin{aligned}
\omega(b) & =\omega(0)(1-i b)^{1 / 3} \\
|\omega(b)| & =|\omega(0)|\left(1+b^{2}\right)^{1 / 6} \\
\angle \omega(b) & =\angle \omega(0)-\frac{1}{3} \tan ^{-1} b .
\end{aligned}
$$

Aside from a slight increase in its length, the principal 


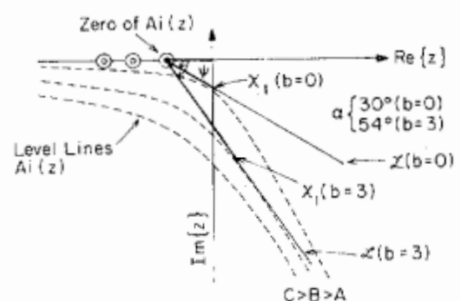

(a)

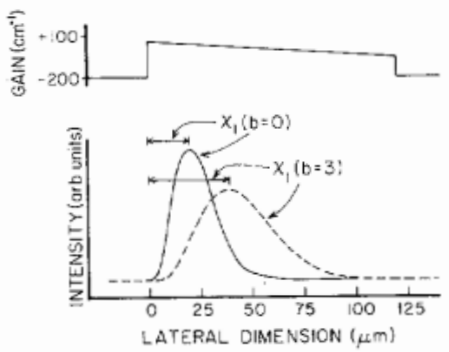

(b)

Fig. 10. (a) Effect of the anti-guiding parameter $b$ on the path of the argument to the Airy function throughout the complex plane. (b) Effect of the anti-guiding factor on the near-field patterns.

effect on $\mathscr{L}$ is a clockwise rotation about $-r_{\nu}$ of $1 / 3$ $\tan ^{-1} b \mathrm{rad}$. The expression for the eigenvalues on the $( \pm)$ branch becomes slightly more complicated:

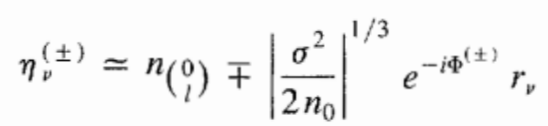

where $\Phi^{( \pm)}=2 / 3 \tan ^{-1} b \pm \pi / 3$. The mode discrimination for the $( \pm)$ branch becomes

$$
\begin{aligned}
\Delta \gamma_{\nu}^{( \pm)}(b)= & \left(1+b^{2}\right)^{1 / 3} \\
& \cdot\left[\cos 2 \varphi \pm \frac{1}{\sqrt{3}} \sin 2 \varphi\right] \Delta \gamma_{\nu}^{( \pm)}(0)
\end{aligned}
$$

where $\varphi=1 / 3 \tan ^{-1} b$. When compared with the special case of no index anti-guiding, for $b=3$, the mode discriminations on the $(+)$ branch are increased by about a factor of two. The cluster of modes centered about the middle of the guide (i.e., the (0) branch) is relatively insensitive to the effect of the anti-guiding parameter. As a result the number of modes on the $(+)$ branch actually decreases with increasing $b$, consistent with the notion that index anti-guiding should shift the high-gain modes towards the lower-gain regions of the waveguide.

Qualitatively, the effect of anti-guiding on the near-field patterns may be determined with the aid of Fig. 10(a) and a simple geometrical argument. As $b$ increases from 0 , the angle $\psi$ that $\mathscr{L}$ makes with the real axis increases. As a result, $\mathscr{L}$ becomes tangent to the level lines of $\operatorname{Ai}(z)$ at a point further removed from $x=0$, implying that the position $\chi_{\nu}$ of the maximum intensity of $E$ has shifted towards the low gain side of the waveguide. Furthermore, as these latter level lines are less strongly curved than those near the origin, the width of the mode increases as well. Near-field profiles along the two lines of $\mathcal{L}(b=0)$ and $\mathfrak{L}(b=3)$ in Fig. 10(a) are compared in Fig. 10(b).

A quantitative generalization of the technique used earlier to determine the near-field and far-field patterns is in- cluded as an Appendix. At this point we simply quote the relations for position of the near-field peak $\chi_{\nu}(b)$

$$
\chi_{\nu}(b)=\frac{2}{\sqrt{3}} \frac{1}{\left(1+b^{2}\right)^{1 / 6}} \frac{\sin (\theta+\pi / 3)}{\cos (\theta+\varphi)} \chi_{\nu}(0)
$$

half-width of the near-field distribution $w_{p}(b)$

$$
w_{\nu}^{2}(b)=\frac{\sqrt{\frac{2 \sin (\pi / 6+\varphi)}{\cos (\theta+\varphi)}}}{\left(1+b^{2}\right)^{1 / 3} \cos (2 \varphi+\theta / 2)} w_{\nu}^{2}(0)
$$

and far-field emission angle $\theta_{v}(b)$

$$
\Theta_{\nu}(b) \simeq\left(1+b^{2}\right)^{1 / 2} \sqrt{\frac{2 \sin (\pi / 6+\varphi)}{\cos (\theta+\varphi)}} \Theta_{\nu}(0)
$$

all expressed in terms of their values in the no anti-guiding case. The angles $\varphi$ and $\theta$ are functions of $b$ defined as

$$
\begin{aligned}
\varphi & =\frac{1}{3} \tan ^{-1} b \\
\tan \theta & =3 \tan \varphi .
\end{aligned}
$$

For example, when $b=3, w_{v}$ increases by a factor of 2 , $\chi_{\nu}$ by a factor of 3 , and $\Theta_{\nu}$ by a factor of 4 .

When the anti-guiding factor is included, the far-field beamwidth remains approximately constant because the increase in the width of the near-field is offset by an increase in the phase curvature. The major effect of antiguiding on the far-field patterns is to shift $\Theta_{v}$ to larger angles. $\Theta_{v}$ is a sensitive function of $b$, and therefore knowledge of the guide parameters (made possible via the halftone process described earlier) allows estimation of the anti-guiding factor.

To make an experimental determination of the anti-guiding factor it is necessary to estimate the gain gradient $s$ defined in (8). The constant $\Gamma_{0}$ is fixed by the requirement that at threshold the modal gain of the lasing mode $\gamma_{1}^{(+)}$ must precisely equal the sum of losses, which are principally due to the mirrors, and are typically about $40 \mathrm{~cm}^{-1}$ for a device $250 \mu \mathrm{m}$ long. In an asymmetric tailored gain halftone laser in which the fraction of injecting contact varies between 100 percent at the left edge of the laser and 0 percent at the right edge, light will be emitted only where the gain is greater than zero. We can estimate the position of transparency by examining the spontaneous emission profile just below threshold. Together with $\Gamma_{0}$, this determines the gain gradient. For example, we estimate the gain gradient in the device of Fig. 1 to be 1 $\mathrm{cm}^{-1} / \mu \mathrm{m}$.

Equation (44) may then be used to calculate the position of the off-axis far-field beam position $\Theta_{1}^{(+)}$as a function of the antiguiding factor $b$. Fig. 11 plots the theoretically expected emission angles for several values of the antiguiding factor along with experimental data from halftone asymmetric tailored gain lasers with differing gain gradients. We find a value $b=2.5 \pm 0.5$, which is in agreement with earlier published results [14]. 


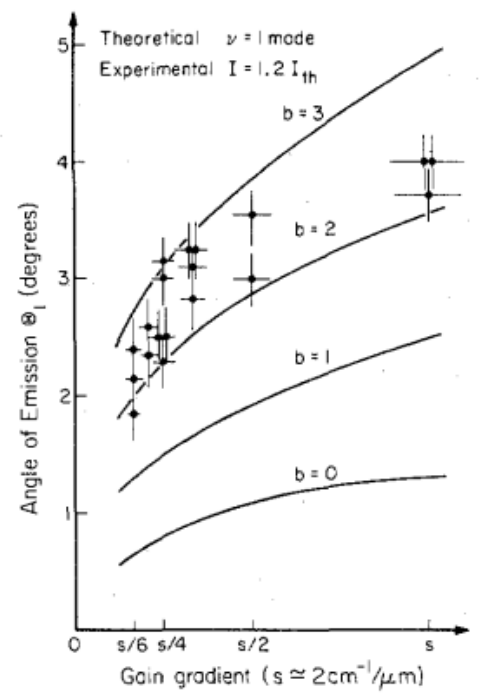

Fig. 11. Plot of the beam emission angle $\theta$ as a function of the anti-guiding parameter $b$ and spatial gain gradient. The experimental data points fit $b$ $=2.5$, in agreement with previously published results.

\section{CONCLUSION}

In conclusion, we have demonstrated a linear tailored gain broad area laser capable of emitting a few hundred milliwatts of optical power into a single, nearly diffraction limited beam only a few degrees wide. We analyzed the lateral optical eigenmodes of such lasers in terms of the Airy function of complex argument, and showed that, unlike uniform phased arrays of semiconductor lasers, the fundamental mode in a tailored gain laser is the lasing mode at threshold, thus making possible the narrow, single-lobed far-field patterns. The mode discrimination between the fundamental and higher order modes may be controlled by variation of the spatial gain gradient, and varies sublinearly with it. We also showed that, unlike all real index-guided lasers or symmetric gain-guided lasers, the higher-order modes of asymmetric tailored gain lasers do not have nulls in their near-field patterns, and that the corresponding far-field patterns are all asymmetric, single-lobed, and only slightly displaced from the fundamental. Finally, we made use of halftone asymmetric tailored gain lasers to estimate the anti-guiding factor.

\section{APPENDIX}

To calculate the near-field and far-field patterns when real index anti-guiding is included, we once again first calculate $\chi_{\nu}$, the position of the peak electric field intensity within the guide. We determine the point at which $\mathscr{L}$ becomes tangent to the level lines of $\operatorname{Ai}(z)$, and rotate the coordinate system so that a Taylor expansion may be used to evaluate the field distribution. When anti-guiding is present $(b \neq 0), \chi_{\nu}$ no longer lies on the anti-Stokes line. The exact evaluation of the level lines of $\operatorname{Ai}(z)$ yield complicated expressions, so we approximate them in the rotated $u-v$ coordinate system by a family of hyperbolas:

$$
u^{2}-\frac{v^{2}}{3}=c^{2}
$$

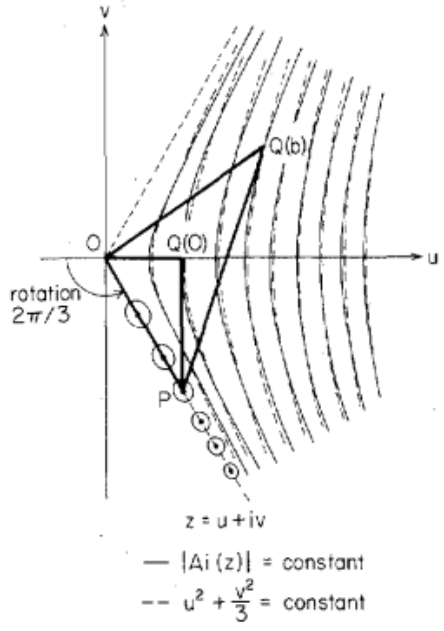

(a)

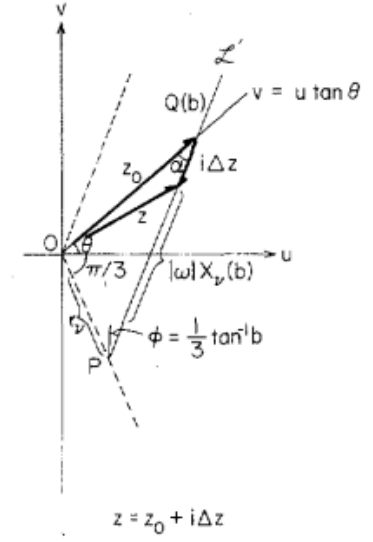

(b)
Fig. 12. (a) Hyperbolic approximation to level lines of $\mathrm{Ai}(z)$ in the rotated coordinate system. (b) Geometric construction for approximation to near-field patterns.

where $c^{2}$ is a positive real constant. This family is centered at the origin, and is asymptotic to $(u / v)= \pm \sqrt{3}$. Fig. 12(a) plots the level lines of $\operatorname{Ai}(z)$ (solid curves) superimposed upon this family of hyperbolas (dashed curve) which shows that, although the approximation is a good one, it may be slightly improved by a small empirical adjustment to the factor 3 in (A.1).

Along with this family of hyperbolas, Fig. 12(a) plots $\mathcal{L}^{\prime}$, the path of the argument of the Airy function in the rotated coordinate system. Fig. 12(b) shows the geometrical relationships among the various quantities more clearly. The equations for the lines $\mathcal{L}_{\nu}^{\prime}$ are

$$
u=u_{v}+m v
$$

where $u_{v}$ is a constant depending on the mode index $\nu$ through the root $-r_{\nu}$ of the Airy function. The slope of this line $m$ is given by

$$
m=\tan \varphi=\tan \left(\frac{1}{3} \tan ^{-1} b\right) .
$$

This line $\mathscr{L}_{\nu}^{\prime}$ is tangent to a level line of the rotated $\operatorname{Ai}(z)$ when the two slopes in the $(u, v)$ plane are equal. From (A.1), we have

$$
\frac{d u}{d v}=\frac{1}{3} \frac{v}{u}
$$

Setting this equal to $m$ in (A.3), we obtain an equation for the locus of points at which the lines $\mathscr{L}_{\nu}^{\prime}$ are tangent to the level lines of the rotated $\operatorname{Ai}(z)$ :

$$
v=-3 \tan \left(\frac{1}{3} \tan ^{-1} b\right) u .
$$

Note that angle $\theta$ in the figure is given by $\theta=\tan ^{-1}[3$ $\left.\tan \left(1 / 3 \tan ^{-1} b\right)\right]$, and that when $b=0, \theta=0$ : in other words, $\mathfrak{L}_{p}^{\prime}(b=0)$ corresponds to the positive real axis (rotated anti-Stokes line) as expected. Furthermore, as $b$ $\rightarrow \infty, \theta \rightarrow \pi / 3$ and $\mathfrak{L}_{\nu}^{\prime}$ becomes asymptotic to the rotated 
Stokes line of $\operatorname{Ai}(z)$. For a typical value of $b=2.5, \theta=$ $51.5^{\circ}$.

Once the tangency point has been located, the geometrical construction shown in Fig. 12(b) may be used to determine $\chi_{\nu} \cdot|\omega| \chi_{\nu}(b)$ is determined by the Sine Law:

$$
\frac{r_{\nu}}{\sin \alpha}=\frac{|\omega| \chi_{\nu}(b)}{\sin \left(\frac{\pi}{3}+\theta\right)} \text {. }
$$

The two angles $\pi / 3+\theta$ and $\alpha=\pi / 2-(\theta+\varphi)$, are known, and so is the side of length $r_{p}$, so that

$$
\chi_{\nu}(b)=\frac{\sin (\theta+\pi / 3)}{\cos (\theta+\varphi)} \frac{r_{\nu}}{|\omega|} .
$$

Note that when $b=0$ we have $\theta=\varphi=0$ and $|\omega| \chi_{\nu}(0)$ $=(\sqrt{3} / 2) r_{\nu}$ as in (26). Using this latter equation, we can therefore rewrite

$$
\chi_{\nu}(b)=\frac{2}{\sqrt{3}} \frac{1}{\left(1+b^{2}\right)^{1 / 6}} \frac{\sin (\theta+\pi / 3)}{\cos (\theta+\varphi)} \chi_{\nu}(0) .
$$

With $b=3, \chi_{\nu}$ increases by a factor of three.

We next determine the near-field pattern $E_{\nu}(x)$ by an expansion about the field maximum $\chi_{v}$. We recall that the path $\mathfrak{L}_{v}^{\prime}$ is defined by $z=\rho+\omega x$ with $0 \leq x \leq \ell$ in the unrotated coordinate system. In the coordinate system which has been rotated by $\pi / 3$, this becomes

$$
z=r_{\nu} e^{-i \pi / 3}+i e^{-i \varphi}|\omega| x
$$

By adding and subtracting the term $i e^{-i \varphi}|\omega| \chi_{\nu}$ we rewrite (A.9) as

$$
\begin{aligned}
z= & {\left[r_{\nu} e^{-i \pi / 3}+i e^{-i \varphi}|\omega| \chi_{\nu}\right] } \\
& +i e^{-i \varphi}|\omega|\left(x-\chi_{\nu}\right)
\end{aligned}
$$

which is of the form

$$
z=z_{0}+i \Delta z
$$

where

$$
\begin{aligned}
z_{0} & =r_{\nu} \frac{\sin (\varphi+\pi / 6)}{\cos (\theta+\varphi)} e^{i \theta} \\
\Delta z & =e^{-i \varphi}|\omega|\left(x-\chi_{\nu}\right) .
\end{aligned}
$$

Observe that for $b=0$ we have $z=u+i v$ as in (30).

The electric field is given near the anti-Stokes line of $\operatorname{Ai}(z)$ by (30) with $u+i v$ replaced by $z_{0}+i \Delta z$. Making the appropriate substitutions and simplifications, it becomes

$E_{\nu}(z)=\frac{1}{2} e^{-i \pi / 6} e^{-(1 / 3) z_{0}^{3 / 2}} \operatorname{Bi}\left(z_{0}\right) \exp \left[-\frac{\left(\Delta z-2 i z_{0}\right)^{2}}{4 z_{0}^{1 / 2}}\right]$

and since $z_{0}$ is independent of $x$ all the lateral-field behavior is contained in the last factor of (A.13). For the case $b=0$, both $z_{0}$ and $\Delta z$ are real, leading to a Gaussian field distribution with a linear phase variation as given by (32). In the general case, $z_{0}$ and $\Delta z$ are complex, leading to a cross coupling between the magnitude and phase terms. However, we may still obtain meaningful insight into the antiguiding case by examining the "magnitude" and "phase" terms individually.

The phase term corresponding to $\phi_{\nu}\left(x-\chi_{\nu}\right)$ of (33) is, from (A.13)

$$
z_{0}^{1 / 2} \Delta z=|\omega| \sqrt{\frac{\sin (\pi / 6+\varphi)}{\cos (\theta+\varphi)}} r_{\nu} e^{-i(\varphi-\theta / 2)}\left(x-\chi_{\nu}\right) .
$$

Equation (A.14) is essentially real because the angle ( $\varphi$ $-\theta / 2$ ) is zero for $b=0$ and $\infty$, rising to only about $5^{\circ}$ in between. When $b=0,(\mathrm{~A} .14)$ reduces to

$$
z_{0}^{1 / 2} \Delta z=|\omega|\left[\left(\frac{r_{v}}{2}\right)^{1 / 2}\left(x-\chi_{\nu}\right)\right] .
$$

It can be shown that the correction term $\left(-1 / 2 r_{v}\right)$ of (33) for the case $b=0$ has an associated obliquity factor $\cos (\theta+\varphi)$ which is very small in the range $b=2$ to 3 , and is therefore neglected. We can therefore express the phase gradient $\phi_{\nu}(b)$ in terms of $\phi_{\nu}(0)$ approximately as

$$
\phi_{\nu}(b) \simeq\left(1+b^{2}\right)^{1 / 2} \sqrt{\frac{2 \sin (\pi / 6+\varphi)}{\cos (\theta+\varphi)}} \phi_{\nu}(0) .
$$

From (36) we see that the beam angle increases by about the same amount. For example, taking $b=3$, the ratio $\Theta_{\nu}(b) / \Theta_{\nu}(0)$ is 3.94 .

The magnitude term corresponding to $\left(x-\chi_{\nu}\right)^{2} / 2 w_{\nu}^{2}$ of (32) is

$$
\frac{\Delta z^{2}}{4 z_{0}^{1 / 2}}=\frac{\left(x-\chi_{\nu}\right)^{2}}{\frac{4}{|\omega|^{2}} \sqrt{\frac{\sin (\pi / 6+\varphi)}{\cos (\theta+\varphi)} r_{\nu}}} e^{-i(2 \varphi+\theta / 2)} .
$$

Of course, it is the real part of (A.17) which determines the width of the near-field distribution in the general case $b \neq 0$. The obliquity factor in this case is quite severe since $2 \varphi+\theta / 2$ approaches $\pi / 2$ quite rapidly as $b \rightarrow \infty$, thereby implying significant broadening of the near-field pattern. We then write the halfwidth of the near-field pattern $w_{\nu}(b)$ as

$$
w_{\nu}^{2}(b)=\frac{\sqrt{\frac{2 \sin (\pi / 6+\varphi)}{\cos (\theta+\varphi)}}}{\left(1+b^{2}\right)^{1 / 3} \cos (2 \varphi+\theta / 2)} w_{\nu}^{2}(0) .
$$

For example, when $b=3$ the near-field pattern broadens by about a factor of two.

Finally, we note that since (A.17) has a significant imaginary part there will be an additional quadratic correction to the linear phase term of (A.15). However, from (A.13) we observe that this correction is of order $\Delta z / 4 z_{0}$. Thus it is not expected to contribute much to the absolute 
angle of beam emission in the far-field, but it will tend to counteract the effect of narrowing the beamwidth due to the increased near-field width.

\section{REFERENCES}

[1] G. H. B. Thompson, "A theory for filamentation in semiconductor lasers including the dependence of the dielectric constant with the injected carrier density," Opto-Electron., vol. 4, pp. 257-310, 1972.

[2] D. R. Scifres, R. D. Burnham, and W. Streifer, "High power coupled multiple stripe quantum well injection lasers," Appl. Phys. Lett., vol. 41 , no. 2, pp. 118-120, July $15,1982$.

[3] D. Ackley, "High-power multiple-stripe injection lasers with channel guides," IEEE J. Quantum Electron., vol. QE-18, pp. 1910-1917, Nov. 1982.

[4] M. Matsumoto, M. Taneya, S. Matsui, S. Yano, and T. Hijikata, "Stable supermode operation in phase-locked laser diode arrays with three index waveguides," J. Appl. Phys., vol. 58, no. 7, pp. 27832785 , Oct. 1, 1985

[5] E. Kapon, J. Katz, and A. Yariv, "Supermode analysis of phaselocked semiconductor lasers," Opt. Lett., vol. 9, pp. 125-127, 1984.

[6] J. K. Butler, D. E. Ackley, and D. Botez, "Coupled mode analysis of phase-locked injection laser arrays," Appl. Phys. Lett., vol. 44, no. 3, pp. 293-295, Feb. 1, 1984.

[7] E. Kapon, C. Lindsey, J. Katz, S. Margalit, and A. Yariv, "Chirped arrays of diode lasers for supermode control," Appl. Phys. Lett., vol. 45 , no. 3 , pp. 200-202, Aug. 1, 1984.

[8] C. Lindsey, Ph.D. dissertation, Calif. Inst. Technol., (Univ. Microfilms Int., Ann Arbor, MI) 1986.

[9] C. P. Lindsey, E. Kapon, J. Katz, S. Margalit, and A. Yariv, "Single contact tailored gain phased array of semiconductor lasers," Appl. Phys. Lett., vol. 45, no. 7, pp. 722-724, Oct. 1, 1984.

[10] C. Lindsey, P. Derry, and A. Yariv, "Fundamental lateral mode oscillation via gain tailoring in a broad area semiconductor laser," Appl. Phys. Lett., vol. 47, no. 6, pp. 560-562, Sept. 15, 1985.

[11] D. F. Welch, D. R. Scifres, P. Cross, W. Streifer, R. D. Burnham, and J. Yaeli, "High power $(575 \mathrm{~mW})$ single-lobed emission from a phased array laser," Electron. Lett., vol. 21, no. 14, pp. 603-604, July 4, 1985 .

[12] D. F. Welch, D. Scifres, P. Cross, H. Kung, W. Streifer, R. D. Burnham, J. Yaeli, and T. L. Paoli, "High power CW operation of a phased array diode laser with diffraction limited output beam," Appl. Phys. Lett., vol. 47, no. 11, pp. 1134-1136, Dec. 1, 1985.

[13] C. Lindsey, P. Derry, and A. Yariv, "Tailored gain broad area lasers with single lobed far-field patterns," Electron. Lett., vol. 21, no. 16 , pp. 671-673, Aug. 1, 1985.

[14] J. Buus, "Principles of semiconductor laser modelling," IEE Proc., vol. 132J, no. 1, pp. 42-51, Feb. 1, 1985.

[15] C. M. Bender and S. A. Orszag, Advanced Mathematical Methods for Scientists and Engineers. New York: McGraw-Hill, 1978, pp. 569570.

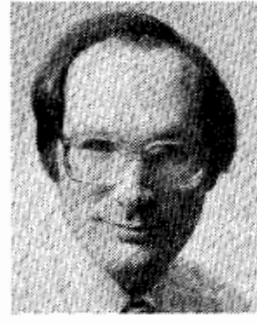

Christopher P. Lindsey received the B.Sc. degree in physics from Harvey Mudd College, Claremont, CA, in 1975.

During the two years following his graduation he organized and administered the computing facilities at Harvey Mudd. From 1977 to 1980 he was employed by Bell Laboratories in Murray Hill, NJ. His research entailed fundamental investigation of the glass transition in polymers using light scattering techniques, principally $\mathrm{Fa}$ bry-Perot and digital autocorrelation. In 1980 he entered the Ph.D. program in applied physics at the California Institute of Technology, Pasadena, CA. His dissertation related to analytical, numerical, and experimental investigations of high-power phased array semiconductor lasers, and he invented methods of tailoring the spatial gain profile within optoelectronic devices, offering a new degree of freedom to the designer of semiconductor lasers.

Dr. Lindsey will be awarded the Ph.D. degree posthumously in June 1987 , having completed all the requirements before his accidental death on August 1, 1986, at the age of 33 .

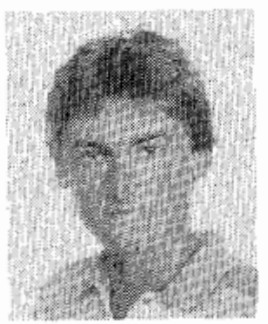

David Mehuys (S'85) was born in Toronto, Ont., Canada, on May 21, 1962. He received the B.A.Sc. degree in engineering science from the University of Toronto in 1984, and the M.S. degree in electrical engineering from the California Institute of Technology, Pasadena, CA, in June 1985.

Currently, he is pursuing the Ph.D. degree in electrical engineering at Caltech, where he is investigating high-power phased array and broad area semiconductor lasers. His related interests include free-space communication, nonlinear laser phenomena, and medical technology.

Amnon Yariv (S'56-M'59-F'70), for a photograph and biography, see p. 399 of the April 1987 issue of this JourNal. 